\author{
Crossing: The INPR Journal \\ Vol. II (2021): 105-109 \\ DOI: $10.21428 / 8766 \mathrm{eb} 43.8 \mathrm{dd} 8584 \mathrm{C}$
}

\title{
Phénoménologie et christianisme : plaidoyers de l'expérience humaine
}

\author{
Francesca Peruzzotti \\ Università cattolica del Sacro Cuore Milano \\ francescaelide.peruzzotti@unicatt.it
}

Lecture de :

Marie-Aimée Manchon, Alentour du verset : Petite phénoménologie des Mystères. Préface d'Emmanuel Falque. Paris : Éditions Ad Solem, «Philosophie », 2019. pp. 536. $26 €$.

Le beau livre Alentour du verset se présente au lecteur comme une œuvre riche et bien dirigée, son caractère intime est le fruit d'un entrelacs de plusieurs niveaux spéculatifs et de nombreuses compétences. C'est en raison de son caractère pluriforme que le lecteur peut y trouver beaucoup de matière à réflexion. Ce livre ne peut en effet pas être réduit à l'une des nombreuses approches possibles de la philosophie de la religion, voire de la théologie philosophique, ni être inclus de manière machinale dans le débat relatif au tournant théologique de la phénoménologie.

Le fil rouge de l'étude se montre très clair, mais il est obtenu par l'autrice à partir de la convocation de plusieurs plans : il s'agit d'un dialogue entre l'écriture sainte et la philosophie en sa version phénoménologique, à travers une méditation savante des Mystères du Rosaire. Cela implique une considération directe de la figure du Christ et aussi de celle de sa mère Marie de Nazareth, les deux portant sur un examen très profitable de l'anthropologie en tant que telle.

Le livre se propose, à travers les analyses qui y sont menées, comme une grande fresque de l'expérience humaine: ni la phénoménologie ni le christianisme ne 
peuvent se situer en dehors de l'expérience, voire se limiter à en tisser ses conditions aprioriques. Dans les deux cas - et dans leur entrelacs qui est ici mis en lumière -, il s'agit de tenter et de renouveler l'approche au vécu, en le laissant s'exprimer, pour tous, à partir des mots fragiles et savants de ceux qui cherchent à donner la parole à la grande aventure humaine.

À ce propos, on doit aussi considérer que les réflexions proposées dans ce livre sont issues du milieu universitaire et de la pratique didactique, mais qu'elles laissent résonner une pensée savante obtenue par une évidente plongée dans la réalité vivante ; la pratique n'est pas mise entre parenthèses au profit de la pensée, mais dans une abondance intellectuelle et existentielle ; l'une féconde l'autre, donnant lieu à un véritable cercle vertueux.

Les considérations suivantes chercheront à s'introduire dans cet élan théorique pour signaler quelques pistes ultérieures par rapport aux choix de fond qui ont été faits, surtout concernant les dimensions épistémologiques. Cela est autorisé par le texte - même s'il ne présente que quelques rapides références à la dimension méthodologique, par rapport à l'entrée dans la description phénoménologique en tant que telle ; l'autrice révèle ses choix dans le discours en train de se faire, en laissant que la réalité se montre en se donnant, ce qui est un motif d'appréciation additionnel.

Tout d'abord, il faut considérer le positionnement de ce livre par rapport à la liaison entre le fait religieux et la phénoménologie. Durant les trois dernières décennies, il a assumé un poids très pertinent, surtout dans les débats francophones. Il ne s'agit pas de la simple alternative entre l'acceptation ou le refus de la rencontre, mais d'une considération plus précise des prises de position explicitées par Manchon.

À partir d'un seul choix théorique, le livre semble atteindre deux objectifs, l'un touchant la philosophie, l'autre la théologie - ou, pour ainsi dire, la réflexion sur l'expérience chrétienne. Il s'agit en effet d'un dialogue entre les versets bibliques et les théories phénoménologiques, une conversation où la pensée théologique en tant que telle - classique, voire contemporaine - s'en trouve presque exclue, sauf par de rapides références aux Pères de l'Église et à Hans Urs von Balthasar, à FrançoisXavier Durrwell et à Giovanni Cesare Pagazzi. La recherche exégétique se révèle, elle aussi, absente, au moins des citations formelles ; au contraire, on s'adresse plusieurs fois à la logique de l'expérience chrétienne des maîtres de spiritualité, à partir des classiques comme François d'Assise et Ignace de Loyola jusqu'au contemporain Pierre-Marie Delfieux.

Si le résultat est positif en ce qui concerne la philosophie, qui se montre capable et appropriée pour élargir ses compétences au domaine du religieux et en particulier de l'expérience chrétienne, cela est tout à fait radical de manière négative en ce qui concerne la théologie : est-ce qu'elle est désormais incapable de travailler son objet dans l'époque contemporaine ? Peut-être la réponse est-elle affirmative, ou 
éventuellement s'agit-il de provoquer la théologie à assumer de façon explicite la méthode phénoménologique ${ }^{1}$. L'autrice se montre cependant plus bienveillante, en assurant que "philosophie et théologie en ce sens ne sont pas deux domaines à opposer, mais deux manières complémentaires d'aborder le même mystère du sens de la vie, par en-bas ou par en-haut, par alentour ou par au-delà » (44). Cela insère ce livre dans le plus grand débat concernant le statut épistémologique de la théologie, qui, à partir du Principe des principes husserlien et de l'interdit heideggérien formulé en 1927, détermine l'exclusion de la pensée théologique, en raison de l'excès du phénomène qu'elle considère, voire du positum qui la caractérise.

À travers ses analyses, Manchon acquiert la possibilité de considérer la pleine dignité des phénomènes religieux, en tant qu'aspects propres à l'humanité la plus commune et, par contrecoup, elle octroie à la théologie la même tâche qu'à la philosophie, celle de mener une enquête anthropologique. Par contre, on n'arrive pas à comprendre si la théologie peut se considérer en tant qu'intelligence critique de la foi, donc si elle peut ambitionner une relevance spéculative pareille à celle philosophique ; si son départ d'en haut barre sa dignité dans la pensée, donc si son universalité est empêchée par son renvoi à la foi. En effet, même si, dans cette visée, phénoménologie et théologie ne sont pas opposées, il demeure que la théologie réside dans une réserve accessible au seul croyant ; et ce, parce que, à celui qui ne partage pas les yeux de la foi, il est empêché de considérer le mystère de la vie comme le croyant peut le faire.

De là découle une dernière question, qui insiste sur les deux domaines : le mérite du livre est celui de montrer, à travers l'aide obtenue par beaucoup d'analyses phénoménologiques (qui ne sont pas toutes l'expression de philosophes qui ont revendiqué une appartenance croyante, ou chrétienne), que les dimensions structurales de l'expérience chrétienne ne sont pas du tout une diminution par rapport à l'expérience humaine en tant que telle ; et qu'au contraire, elles livrent sa mise à l'épreuve, jusqu'à fournir sa démesure en tant que sa forme la plus adéquate. Si cela est un profit considérable pour la pensée du christianisme, il s'ouvre à un risque quand il entre dans le débat plus vaste : faire du christianisme seulement une narration possible, en tant que modèle n'ayant aucune référence avec la réalité, mais demeurant une vision du monde possible, même si non effective. Il ne s'agit pas de refuser cette approche, mais de signaler le désir pour une ultérieure prise de position de son autrice, pour que ses recherches puissent sortir de ce cercle très clos et montrer la spécificité décisive propre à ce qui est gardé par les Écritures, pour ne pas les réduire à un conte, efficace, mais vide de réalité comme un conte édifiant.

\footnotetext{
${ }^{1}$ Nous nous référons en particulier à l'espoir adressé aux théologiens de « lire phénoménologiquement les événements de révélation consignés dans les Écritures », formulé dans J.-L. Marion, De surcroît, PUF, Paris 2001, p. 34. Le livre de Manchon arrive de manière complète à répondre à ce vœu.
} 
C'est sur le statut de l'Écriture et son rôle que porte la deuxième tranche de questions qui sont ouvertes par le livre. Encore une fois, c'est à partir de la phénoménologie que Manchon définit la légitimité de son approche à la Bible. En particulier, c'est Emmanuel Levinas qui est convoqué au point de départ en tant que garant de la rigueur du mélange. L'originalité de l'autrice est cependant tout de suite revendiquée, en affirmant que le fait de laisser que les paroles de la philosophie résonnent à partir de celles de la Bible est dû au « principe d'incarnation qui s'impose à toute lecture chrétienne de l'Écriture » (43).

L'immersion dans la chair et dans la profondeur du monde se révèle du fil rouge à partir duquel le livre se déploie : les mystères de la vie du Christ et de Marie proposés par le chapelet. Cela donne tout de suite aux versets bibliques la couleur des tonalités émotives propres à l'expérience humaine, que Manchon décline et précise à partir des couronnes issues de la tradition. C'est le fait même de choisir le chapelet à empêcher une réduction savante de la Bible. Il assure le déploiement complet de la sagesse biblique au cœur de l'expérience de la vie chrétienne la plus commune, comme il est arrivé tout au long les derniers siècles et les communautés ecclésiales les plus différentes.

Cela n'est pas sans une évidente acribie ainsi qu'une connaissance profonde de la Bible en tant que telle, que ses versets se répondent dans un dialogue très dense tout au long de l'écrit, claire confirmation de l'adage selon lequel «Scriptura sui ipsius interpres ». Le mérite de Manchon - preuve qu'elle a tiré une excellente leçon de Jean-Louis Chrétien (ce n'est pas par hasard qu'il est le philosophe le plus cité dans le texte) - provient de ce qu'elle a su mener jusqu'à son terme ce que le principe mentionné implique. Le fait que la Bible s'interprète par elle-même - qu'elle résonne à partir de ses liens intérieurs, l'ouvre à l'extérieur, donc implique une résonance et une polyphonie des voix historiques qui devient nécessaire. La convocation de tous les philosophes, qu'ils soient ou pas harmoniques avec la référence à l'Évangile qu'ils contribuent à mettre à jour, se propose comme l'un des possibles plongés dans la concrétude de l'époque contemporaine.

Toutefois, il faut en même temps souligner que ce procès s'expose au risque déjà signalé, celui de perdre la relevance théorique spécifique de l'écrit biblique. En effet, la parcellisation des versets risque d'exposer l'interprétation à la perte de la vision d'ensemble, à la possibilité de définir de manière explicite quel est le principe herméneutique global qui lie le Premier avec le Nouveau Testament et leurs livres entre eux, à partir d'une considération plus profonde du principe christologique qui le soutient.

La possibilité de dégager le lien indissociable des aspects propres de la vie en tant que telle à partir de ce que le Christ a vécu est en effet accessible à partir de l'explicitation de sa propre singularité trinitaire. 
On peut conclure cette brève recension uniquement en répétant que cette œuvre est précieuse parce qu'elle déploie beaucoup de contenus et d'analyses phénoménologiques tout à fait pertinents dans le domaine anthropologique et religieux. En même temps, sa lecture donne à envisager de manière nouvelle l'activité humaine tout à fait particulière qu'est la pensée en tant que telle, dont philosophie et théologie sont deux des expressions majeures. 